\title{
Pigeon adenovirus and pigeon torque teno virus associated with acute multifocal hepatic necrosis in pigeons in Queensland, Australia
}

\author{
Kalpana Agnihotri ${ }^{1}\left[\right.$ Craig Smith $^{1} \cdot$ Jane Oakey $^{1} \cdot$ Greg Storie $^{1}$
}

Received: 23 November 2020 / Accepted: 23 January 2021

(c) Crown 2021

\begin{abstract}
In 2018, an outbreak resulting in deaths of 28 breeding pigeons was reported north of Brisbane, Australia. The affected birds had runny nasal discharge and poor body condition. Two birds were submitted to Biosecurity Sciences Laboratory, Brisbane, for investigation. A range of diagnostic tests excluded a number of known pathogens, and no virus was isolated in cell culture. Histopathological examination revealed severe acute multifocal necrosis in the liver with eosinophilic intranuclear inclusions in hepatocytes and Kupffer cells. High-throughput sequencing (HTS) revealed full-length sequences for pigeon adenovirus 1 (PiAd-A) and pigeon torque teno virus (PTTV). This report indicates concomitant PiAd-1and PTTV infections in Australian pigeons.
\end{abstract}

Domestic pigeons (Columba livia) are susceptible to a number of viral infections, and those resulting in high morbidity and mortality are of major concern to the meat and racing pigeon industry. Viral infections manifest with a wide spectrum of symptoms, including loss of body weight, respiratory problems, diarrhoea, vomiting, nervous signs, depression and conjunctivitis [1]. Several viruses are known to cause infections in pigeons, including paramyxoviruses, circoviruses, rotaviruses, herpesviruses, poxviruses, and adenoviruses [1]. The clinical signs of these viruses are nonspecific, and sources of these viruses remain unclear.

Adenoviruses (members of the family Adenoviridae) are non-enveloped, double-stranded DNA viruses with a genome size ranging from 26 to $45 \mathrm{kbp}$. Adenoviruses of the genus Aviadenovirus infect birds and have been isolated from chickens, turkeys, geese, ducks, pigeons, and other avian species [2]. Several serotypes of fowl adenoviruses have been isolated from healthy and diseased pigeons [3], and infections with these viruses have been reported to induce diarrhoea and depression in young birds $[4,5]$. Two

Handling Editor: Sheela Ramamoorthy.

Kalpana Agnihotri

Kalpana.Agnihotri@daf.qld.gov.au

1 Biosecurity Sciences Laboratory, Biosecurity Queensland, Department of Agriculture and Fisheries, Queensland Government, Health and Food Sciences Precinct, 39, Coopers Plains, QLD 4108, Australia pigeon-specific adenoviruses have been identified, differing in the clinical form of the disease induced in infected pigeons and gross lesions observed at necropsy. Pigeon aviadenovirus 1 (PiAd-1) infection results in a classical adenovirus disease with inclusion body hepatitis. PiAd-1 infects young pigeons with clinical signs that include diarrhoea, vomiting, and weight loss over a period of approximately one week [4] and causes high morbidity and mortality. These clinical signs have similarities to young pigeon disease syndrome (YPDS); however, other viruses also have been reported to contribute to the aetiology of YPDS [6, 7]. PiAd-1 is serologically distinct from the fowl adenoviruses [5]. The only full-length genome sequence available as a reference sequence, IDA4, has a genome organisation that is the most similar to turkey adenovirus 1 (TAd-1) $[8,9]$.

Pigeon aviadenovirus 2 (PiAd-2) causes necrotizing hepatitis in infected pigeons. It infects pigeons of all ages, causing extensive hepatic necrosis and death $[10,11]$. The genome of PiAd-2 is smaller than and distinct from PiAd-1 [12]. Flocks infected with pigeon adenoviruses have also been reported to have concomitant infections with pigeon circovirus and pigeon herpesvirus [13].

Torque teno viruses (TTVs) are ubiquitous in nature and widely distributed throughout the world. They are nonenveloped, negative-sense, ssDNA viruses with a genome size ranging from 1.5 to $3.8 \mathrm{kbp}$, belonging to the genus Alphatorquevirus of the family Anelloviridae [14]. TTVs have been reported to infect pigeons, and a species-specific pigeon TTV (PTTV) was first reported in China in 2012 and 
is considered prevalent in pigeons from that region. Infected birds display clinical signs such as respiratory signs, weight loss, and diarrhoea [15, 16].

In this report, we describe adenovirus infections in a pigeon flock that also had concomitant infections with pigeon torque teno virus (PTTV). Although most viruses that infect pigeons have a broad host range, both PiAd-1 and PTTV appear to exhibit species specificity.

In 2018, a small hobby farm north of Brisbane (Queensland, Australia) reported an outbreak of disease in breeding pigeons. The owner had sourced the breeding birds from three other farms in Queensland. Out of a total of 50 birds of mixed age on the farm, 28 had died over a period of a few days. Before they died, the birds were reported to have runny nasal discharge and poor body condition. Two moribund or dead pigeons were submitted to the Biosecurity Sciences Laboratory, Brisbane. Bird 1 (P18-05523-6) was submitted dead, and bird 2 (P18-05523-7) was moribund and depressed and was euthanized by cervical dislocation. Necropsy of bird 1 showed it to be in moderate body condition with a swollen and mottled liver. Necropsy of bird 2 revealed increased liquidity of intestinal contents with faecal staining around the vent suggestive of diarrhoea. Tracheal and cloacal swabs from both birds were collected separately into inhouse viral transport medium supplemented with $10 \mathrm{mg}$ of bovine albumin, $100 \mathrm{IU}$ penicillin, and $100 \mu \mathrm{g}$ of streptomycin and fungizone per mL. Fresh tissue samples, including gastrointestinal tract and liver samples, were collected for bacteriology, virology, and parasitology investigation. The bacterial culture from both liver tissue samples produced no significant aerobic growth, and no Salmonella spp. were isolated. The gastrointestinal tract was examined for coccidia and helminths by microscopy. The gastrointestinal tract of bird 2 showed moderate numbers of Eimeria spp. coccidia, identified as E. labbenna and E. columbarum based on the oocyst size. Liver samples from both birds tested negative by PCR for Chlamydiaceae and pigeon herpesvirus. The cloaca and trachea from both birds tested negative by PCR for pigeon paramyxovirus, pigeon rotavirus, Newcastle disease virus, and influenza A virus. Liver samples from both birds were used for virus isolation in chicken embryonic fibroblast cells, and no virus was isolated in cell culture.

Tissue samples from liver, lung, kidney, and heart were collected separately in $10 \%$ neutral buffered formalin. Formalin-fixed tissue sections were stained with haematoxylin and eosin for histological examination. Histologically, the liver from bird 1 showed severe acute multifocal necrosis (Fig. 1A). There were eosinophilic intranuclear inclusions present in hepatocytes and Kupffer cells (Fig. 1B). The kidney showed moderately severe, subacute, multifocal, lymphoplasmacytic and heterophilic interstitial nephritis. The lung and heart showed no abnormalities. Histological examination of the lung, liver, kidney, and heart tissues of bird 2 revealed no abnormalities.

Liver samples from both birds were used for highthroughput sequencing (HTS). Total DNA was extracted using a QIAGEN DNeasy® 96 Blood and Tissue Kit (QIAGEN Pty Ltd.), following manufacturer's instructions, with a final elution volume of $30 \mu$. The DNA concentration was measured using a Qubit Fluorometer and a Qubit dsDNA HS Assay Kit (Thermo Fisher Scientific). Libraries (differentially indexed) were prepared using a Nextera XT DNA Library Preparation Kit (Illumina) following the manufacturer's instructions. The size and purity of the pooled sequence library was quantified using a 2200 TapeStation System (Agilent). The library was sequenced on a NextSeq 500 Sequencing Platform using a NextSeq Mid Output Kit v2 (Illumina), producing paired reads $150 \mathrm{bp}$ in length.

Indexing quality control and FASTQ file generation were initially performed using a BaseSpace Sequence Hub (Illumina), with additional trimming performed using the Geneious ${ }^{\circledR}$ version 11.0.3 (Biomatters) plugin BBDuk [17]. Continuing with the Geneious ${ }^{\circledR}$ assembler, de novo assembly was performed on the 4.8 million and 5.3 million paired
Fig. 1 Pigeon (Columba livia) liver (haematoxylin and eosin). (A) Severe acute multifocal necrosis in hepatic tissue. Scale bar, $200 \mu \mathrm{m}$. (B) Eosinophilic intranuclear inclusions in hepatic cells. Scale bar, $20 \mu \mathrm{m}$
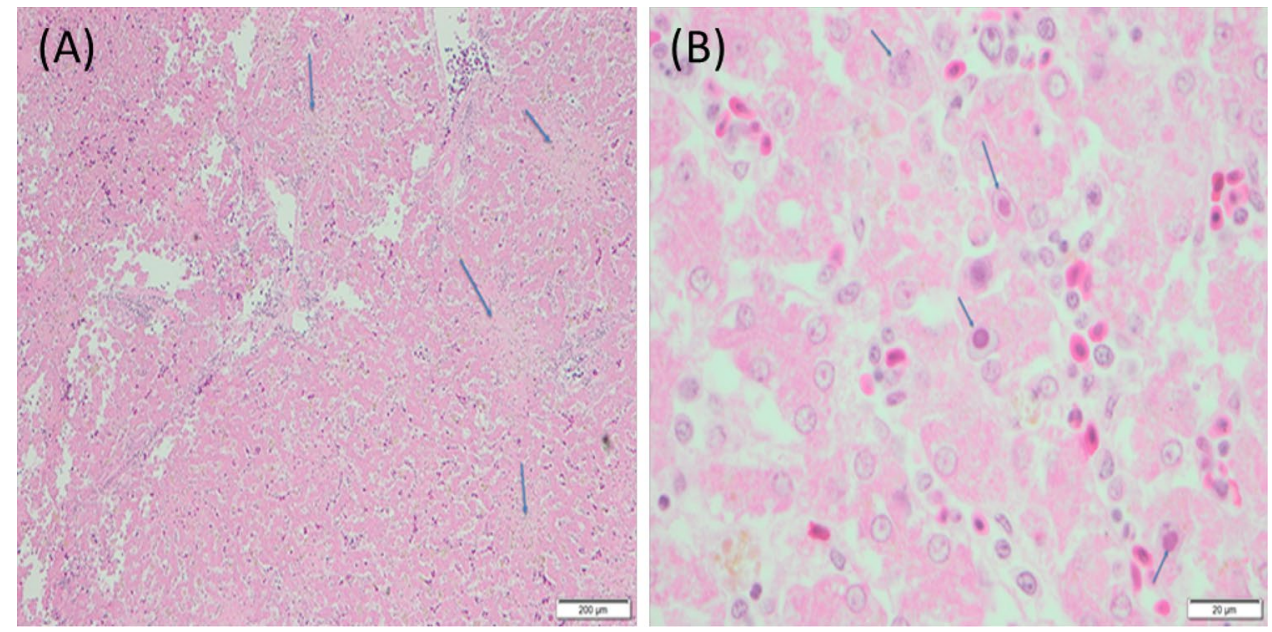
reads from the respective samples, and sequence homology searches of the resulting contigs were performed using GenBank (NCBI) Basic Local Alignment Search Tool (BLAST) against the CoreNucleotide collection (http://www.ncbi.nlm. nih.gov/genbank).

HTS analysis (de novo) of the liver sample from bird 1, the pigeon with severe acute hepatic necrosis, produced contigs partially aligning to the genome sequence of PiAd1(IDA4, NC_024474). No PiAd-1-like contigs were identified in the liver sample from bird 2. To improve coverage, the data from bird 1 were mapped to the reference strain IDA4 using Geneious. A complete genome sequence of PiAd-1 (45,476 bases) was obtained using 23,833 reads (less than $1 \%$ of total reads) and showed a high level of sequence identity (99.3\%) to the reference strain. The sequence was submitted to the GenBank database with the accession number MW286325. The full genome sequence was annotated using the online tool ORF Finder (https://www.ncbi.nlm. nih.gov/orffinder/). The open reading frames (ORFs) were subjected to a BLASTp homology search, and results with high scores of homology to the IDA4 annotations were used to mark the regions in the genome, as presented in Table 1. Most of the genes showed the highest sequence similarity to those of the reference sequence IDA4, and they had the same order and orientation. The central part of the genome containing 16 consecutive genes that correspond to proteins IVa2 to pVIII has been reported to be conserved in all adenoviruses [18]. This region, along with the fiber-1 gene, is conserved across all aviadenovirus sequences studied to date [19]. The two overlapping fiber genes with a known frameshift, annotated as fiber- 1 and fiber-2, have $99.6 \%$ and $98 \%$ amino acid sequence identity to those of the reference sequence IDA4 [9]. The gene encoding the $\mathrm{U}$ exon protein, located between the pVIII and fiber-1 genes on the complementary strand and present in almost all adenoviruses, is also conserved in the PiAd-1 sequence.

The full-length nucleotide sequence of PiAd- 1 from bird 1 was compared in a multiple sequence alignment with previously reported full-length adenoviral sequences, using online MAFFT version 7.0 [20-22]. The MAFFT-generated alignment was used to construct a phylogenetic tree using the neighbour-joining method and the Jukes-Cantor substitution model with 1000 bootstrap replicates. The other aviadenovirus sequences used in the phylogenetic analysis were representative sequences from pigeon (NC024474, NC031503), duck (Y09598), turkey (NC022612, GU936707, KF477313 and NC014564), fowl (KT862805, KT862807, KT862809, KT862810, KT862812, AF083975, GU188428, KC493646, KU342001, MF577036, NC014969, NC038332), goose (JF510462, NC017979), and parrot (KJ675568). The fowl adenoviruses have been studied extensively, and their fulllength genome sequences are available in the database [23-25]. The adenoviral sequence from a parrot (KX577802, not shown in Fig. 2) is the only full-length sequence available in the database representing adenovirus infection in an Australian bird, showed sequence identity of $56.04 \%$ with PiAd-1 [26]. The PiAd-1 sequence from bird 1 clustered in the phylogenetic tree with the full-length genome sequence of pigeon adenovirus A strain IDA4, which had the most sequence similarity (Fig. 2). The results suggest that PiAd-1 is species-specific and that its sequence has remained conserved since its first detection in the Netherlands in 1995 [9]. PiAd-1 has been shown to be closely related to turkey adenovirus 1 (TAd-1) [9], and the complete genome sequence of PiAd-1 from bird 1 was found to be $79.30 \%$ identical to that of TAd-1 but differed from those of turkey adenoviruses 4 and 5 .

Similar analysis of the HTS data from the liver samples from both pigeons identified contigs corresponding to the genome sequence of pigeon torque teno virus (PTTV). A total of 2928 and 5814 reads from the respective samples were mapped to the reference sequence of PTTV. The reads from both birds were each mapped to the complete genome sequence of KF477316, and 1656 bases corresponding to PTTV sequences were identified. The sequences were submitted to the GenBank database with accession numbers MW286326 and MW286327. The ORFs encoding the Rep and Cap proteins were found to be conserved in both sequences. ORF1 encodes the replication (Rep) protein, from nucleotides 915 to 1385 in the genome, encoding 156 amino acids. ORF2 encodes the capsid (Cap) protein on the reverse strand from nucleotides 660 to 1121 in the genome, encoding 153 amino acids. The PTTV sequences from birds 1 and 2 showed a high degree of sequence similarity (97.54\% and $96.97 \%$ identity, respectively) to PTTV sequences reported from China (GenBank accession no. KF477316).

The full-length nucleotide sequence of PTTV was compared in a multiple sequence alignment to known full-length torque teno virus sequences, using online MAFFT version 7.0 [20-22]. The MAFFT-generated alignment was used to construct a phylogenetic tree using the neighbour-joining method and the Jukes-Cantor substitution model with 1000 bootstrap replicates. The other sequences used in the phylogenetic analysis included representative sequences of PTTV from China (MF576433, MF576434, MF576435, KF477315, KF477316, KF477317, KF477318, KF477319 and KF372027), pigeon circovirus (PiCV) (EF394777, AY754015), chicken anemia virus (CAV) (M55918, JX964755, JX260426), torque teno felis virus (TTFV) (HM142588, HM142589), human torque teno mini virus (TTmV) (NC014095, NC014097), and torque teno virus (TTV) (NC014480, NC015783, GU376737, JF937658, JF937661, AY823991). The tree indicates a monophyletic lineage of pigeon-specific torque teno viruses, as both PTTV sequences from Australia clustered with the PTTV 
Table 1 Predicted open reading frames in the PiAdV-1 genome, showing the reading frame and strand, nt position, number of amino acids (aa) in the encoded protein, and percent sequence identity to the reference genome IDA4

\begin{tabular}{|c|c|c|c|c|}
\hline Gene/ORF & $\begin{array}{l}\text { Reading } \\
\text { frame-strand }\end{array}$ & nt position & No. of aa & $\begin{array}{l}\text { Percent identity } \\
\text { to IDA4 reference } \\
\text { strain }\end{array}$ \\
\hline Protein ORF52 & $1-\mathrm{N}$ & $1445->411$ & 344 & $99.7 \%$ \\
\hline Protein ORF1 & $2-\mathrm{P}$ & $2201->2866$ & 221 & $98.3 \%$ \\
\hline Protein ORF2 & $3-\mathrm{P}$ & $3447->4259$ & 270 & $98.9 \%$ \\
\hline Protein ORF14A & $3-\mathrm{N}$ & $5019->4294$ & 241 & $98.9 \%$ \\
\hline Protein ORF14 & $2-\mathrm{N}$ & $6163->4853$ & 436 & $98.9 \%$ \\
\hline Protein ORF13 & $1-\mathrm{N}$ & $6554->5727$ & 275 & $99.6 \%$ \\
\hline Protein ORF12 & $3-\mathrm{N}$ & $7911->6619$ & 430 & $99.7 \%$ \\
\hline Encapsidation protein IVa2 & $2-\mathrm{N}$ & $8833->7511$ & 439 & $99.6 \%$ \\
\hline DNA polymerase & $1-\mathrm{N}$ & $12596->8817$ & 1259 & $99.3 \%$ \\
\hline $52 \mathrm{~K}$ encapsidation protein & $2-\mathrm{P}$ & $14627->15808$ & 393 & $100 \%$ \\
\hline Terminal protein precursor $\mathrm{pTP}$ & $2-\mathrm{N}$ & $14725->12578$ & 715 & $99.7 \%$ \\
\hline Capsid protein precursor pIIIa & $3-\mathrm{P}$ & $15795->17552$ & 585 & $99.7 \%$ \\
\hline Capsid protein III & $2-\mathrm{P}$ & $17210->19339$ & 709 & $99.6 \%$ \\
\hline Core protein precursor $\mathrm{pVII}$ & $3-\mathrm{P}$ & $19353->19601$ & 82 & $100 \%$ \\
\hline Core protein precursor $\mathrm{pX}$ & $1-\mathrm{P}$ & $19687->20280$ & 197 & $99.5 \%$ \\
\hline Capsid protein precursor $\mathrm{pVI}$ & $1-\mathrm{P}$ & $20359->21003$ & 214 & $100 \%$ \\
\hline $\begin{array}{l}\text { Capsid protein II involved in capsid } \\
\text { morphogenesis }\end{array}$ & $2-\mathrm{P}$ & $21035->23950$ & 971 & $99.4 \%$ \\
\hline Protease & $1-\mathrm{P}$ & $24001->24708$ & 235 & $100 \%$ \\
\hline ssDNA binding protein (DBP) & $2-\mathrm{N}$ & $26179->24782$ & 465 & $99.2 \%$ \\
\hline Hexon assembly protein $100 \mathrm{~K}$ & $1-\mathrm{P}$ & $26926->29649$ & 907 & $99.3 \%$ \\
\hline Encapsidation protein $22 \mathrm{~K}$ & $2-\mathrm{P}$ & $29045->29848$ & 267 & $100 \%$ \\
\hline Protein $33 \mathrm{~K}$ & $3-\mathrm{P}$ & $29271->30110$ & 279 & $96.9 \%$ \\
\hline Capsid protein precursor $\mathrm{pVIII}$ & $2-\mathrm{P}$ & $30158->30931$ & 257 & $100 \%$ \\
\hline Fiber-1 & $3-\mathrm{P}$ & $31029->31934$ & 301 & $99.6 \%$ \\
\hline $\mathrm{U}$ exon-exon encoding $\mathrm{C}$ terminus & $2-\mathrm{N}$ & $31195->30491$ & 234 & $100 \%$ \\
\hline Fiber-2 & $1-\mathrm{P}$ & $31750->34053$ & 767 & $98.0 \%$ \\
\hline Protein ORF22 & $2-\mathrm{N}$ & $34654->34064$ & 196 & $100 \%$ \\
\hline Protein ORF20A & $3-\mathrm{N}$ & $35112->34696$ & 138 & $100 \%$ \\
\hline Protein ORF20 & $1-\mathrm{N}$ & $36341->35382$ & 319 & $100 \%$ \\
\hline ORF19 & $3-\mathrm{N}$ & $41292->36535$ & 1585 & $98.2 \%$ \\
\hline GAM-1/ORF8 & $2-\mathrm{P}$ & $42482->43411$ & 309 & $99.7 \%$ \\
\hline Protein ORF58 & $2-\mathrm{P}$ & $43589->43990$ & 133 & $91.2 \%$ \\
\hline Protein ORF58A & $3-\mathrm{P}$ & $44082->44456$ & 124 & $100 \%$ \\
\hline Protein ORF59 & $3-\mathrm{P}$ & $44532->44933$ & 133 & $96.2 \%$ \\
\hline Protein ORF60 & $1-\mathrm{P}$ & $45115->45450$ & 111 & $97.2 \%$ \\
\hline Protein ORF61 & $3-\mathrm{N}$ & $42597->42247$ & 116 & $99 \%$ \\
\hline Protein ORF62 & $3-\mathrm{N}$ & $42243->42100$ & 47 & $100 \%$ \\
\hline
\end{tabular}

sequences from China, which had the highest sequence identity (Fig. 3).

In Australia, several disease outbreaks in domestic, feral, and racing pigeons have been reported to cause sickness and death in infected pigeons. The clinical signs and tissue lesions are similar to infection with PiAd-1 and PTTV in this report. The viruses reported to be associated with hepatic lesions are adenoviruses [27], herpesviruses [28, 29], circoviruses [30], and rotaviruses [31, 32]. Whilst both PiAd-1 and PTTV are known to be associated with hepatic lesions, their role in pathogenicity of disease needs further investigation. PTTV is ubiquitous in nature; however, its prevalence in Australian birds has not been determined. HTS allowed identification of these viruses in the infected pigeon flock. This report shows that concomitant infections of both of these viruses can occur and confirms the presence of these viruses in Australian pigeons. This study demonstrates the value of HTS for identification of viruses and other pathogens to improve our understanding of viral prevalence and incidence. 


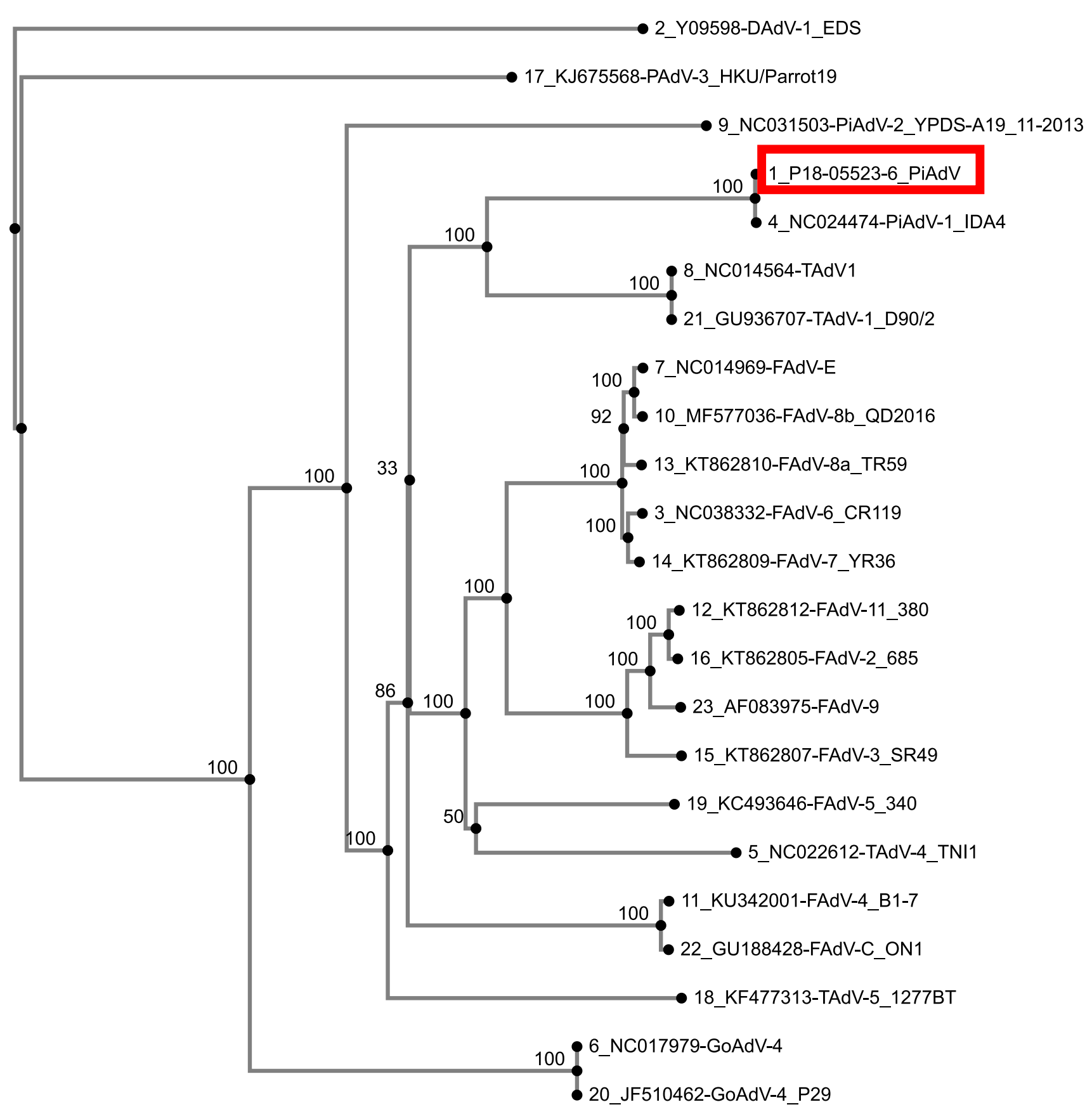

Fig. 2 Phylogenetic tree based on a MAFFT alignment of full-length adenovirus genome sequences, constructed using the neighbour-joining method and the Jukes-Cantor substitution model with 1000 bootstrap replicates. 


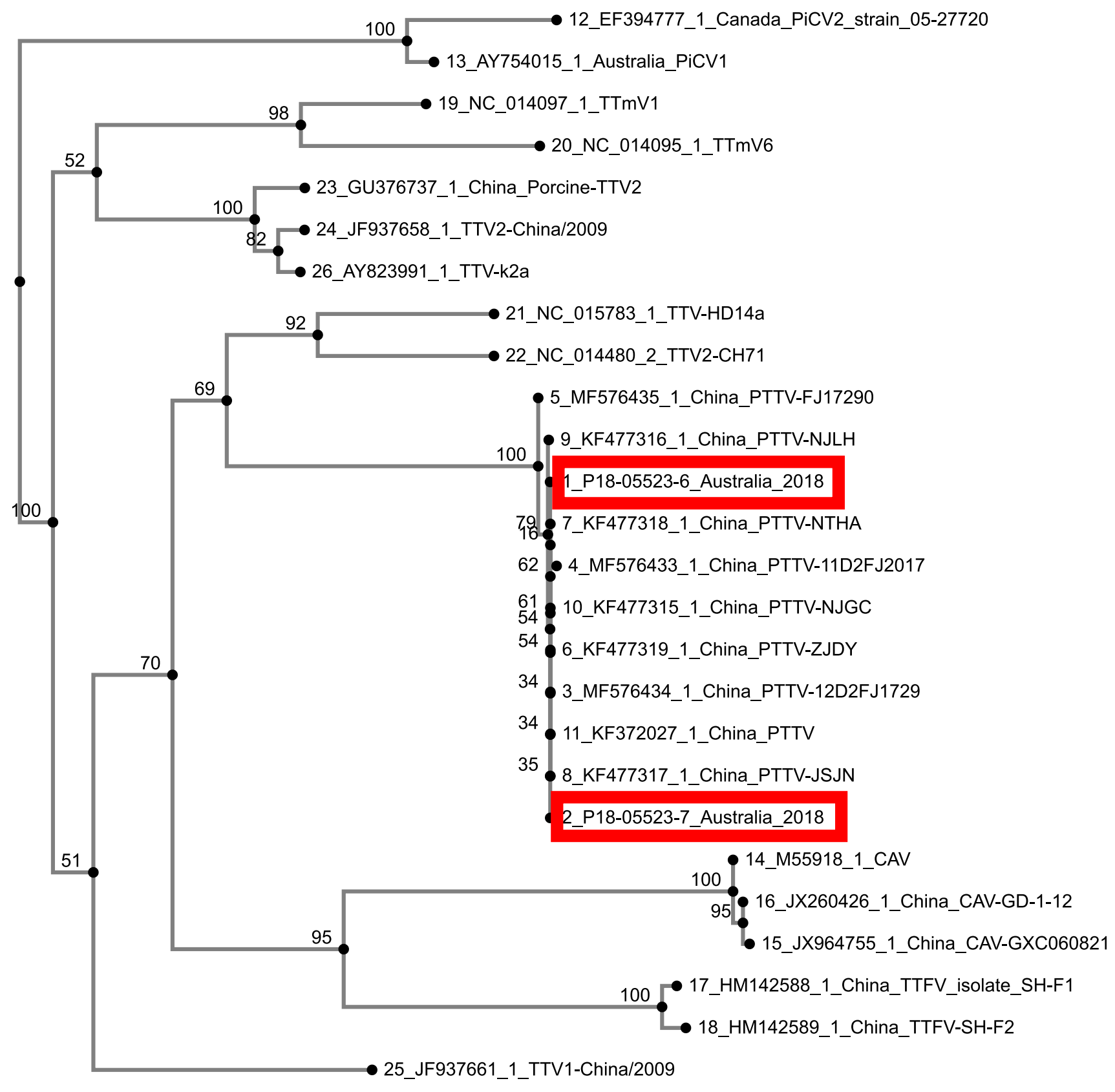

Fig. 3 Phylogenetic tree based on a MAFFT alignment of full-length torque teno virus nucleotide sequences, generated using the neighbourjoining method and the Jukes-Cantor substitution model with 1000 bootstrap replicates.

Acknowledgements We thank Dr. Gavin Wilkie at Illumina for his sequencing expertise and assistance. We thank Darren Underwood, Les Barker, Cath Covacin, and Wafa Shinwari for the laboratory data. We also thank Shirley Turner and Louise Jackson for useful comments in preparation of the manuscript. This work was funded by Biosecurity Queensland, Queensland Department of Agriculture and Fisheries.

\section{References}

1. Marlier D, Vindevogel H (2006) Viral infections in pigeons. Vet J 172(1):40-51
2. Benkö M, Harrach B (1998) A proposal for a new (third) genus within the family Adenoviridae. Adv Virol 143(4):829-837

3. McFerran J, Smyth J (2000) Avian adenoviruses. Rev Sci Tech 19(2):589-598

4. Goryo $\mathrm{M}$ et al (1988) Inclusion body hepatitis due to adenovirus in pigeons. Avian Pathol 17(2):391-401

5. Hess M, Prusas C, Monreal G (1998) Growth analysis of adenoviruses isolated from pigeons in chicken cells and serological characterization of the isolates. Avian Pathol 27(2):196-199

6. Duchatel J-P, Szeleszczuk P (2011) Young pigeon disease syndrome. Med Weter 67(5):291-294 
7. Raue $\mathrm{R}$ et al (2005) A disease complex associated with pigeon circovirus infection, young pigeon disease syndrome. Avian Pathol 34(5):418-425

8. Kaján GL et al (2010) The first complete genome sequence of a non-chicken aviadenovirus, proposed to be turkey adenovirus 1 . Virus Res 153(2):226-233

9. Marek A et al (2014) Complete genome sequences of pigeon adenovirus 1 and duck adenovirus 2 extend the number of species within the genus Aviadenovirus. Virology 462:107-114

10. De Herdt P et al (1995) An epidemic of fatal hepatic necrosis of viral origin in racing pigeons(Columba livia). Avian Pathol 24(3):475-483

11. Vereecken M, De Herdt P, Ducatelle R (1998) Adenovirus infections in pigeons: a review. Avian Pathol 27(4):333-338

12. Teske $\mathrm{L}$ et al (2017) Identification of a novel aviadenovirus, designated pigeon adenovirus 2 in domestic pigeons (Columba livia). Virus Res 227:15-22

13. Stenzel T et al (2012) Epidemiological investigation of selected pigeon viral infections in Poland. Vet Rec 171(22):562-562

14. Biagini P (2009) Classification of TTV and related viruses (anelloviruses), in TT Viruses. Springer, New York, pp 21-33

15. Zhang Z, Dai W, Dai D (2017) Molecular characterization of pigeon torque teno virus (PTTV) in Jiangsu province. Comput Biol Chem 69:10-18

16. Zhang $\mathrm{Z}$ et al (2013) Complete genome sequence of a novel pigeon torque teno virus in china. Genome Announc 1(6):e01076-e1113

17. Kearse $\mathrm{M}$ et al (2012) Geneious Basic: an integrated and extendable desktop software platform for the organization and analysis of sequence data. Bioinformatics 28(12):1647-1649

18. Davison AJ, Benkő M, Harrach B (2003) Genetic content and evolution of adenoviruses. J Gen Virol 84(11):2895-2908

19. Kaján GL et al (2012) Genome sequence of a waterfowl aviadenovirus, goose adenovirus 4. J Gen Virol 93(11):2457-2465

20. Katoh K (2005) MAFFT version 5: improvement in accuracy of multiple sequence alignment. Nucleic Acids Res 33(2):511-518

21. Katoh K, Frith MC (2012) Adding unaligned sequences into an existing alignment using MAFFT and LAST. Bioinformatics 28(23):3144-3146
22. Katoh K, Rozewicki J, Yamada KD (2019) MAFFT online service: multiple sequence alignment, interactive sequence choice and visualization. Brief Bioinform 20(4):1160-1166

23. Joubert HW et al (2014) Molecular differentiation and pathogenicity of Aviadenoviruses isolated during an outbreak of inclusion body hepatitis in South Africa. J S Afr Vet Assoc 85(1):01-08

24. Marek A et al (2013) The first whole genome sequence of a Fowl adenovirus B strain enables interspecies comparisons within the genus Aviadenovirus. Vet Microbiol 166(1-2):250-256

25. Meulemans $G$ et al (2001) Polymerase chain reaction combined with restriction enzyme analysis for detection and differentiation of fowl adenoviruses. Avian Pathol 30(6):655-660

26. Shubhagata $D$ et al (2017) A novel pathogenic aviadenovirus from red-bellied parrots (Poicephalus rufiventris) unveils deep recombination events among avian host lineages. Virolo 502:188-197

27. Ketterer $P$ et al (1992) Inclusion body hepatitis associated with an adenovirus in racing pigeons in Australia. Aust Vet J 69(4):90

28. Boyle D, Binnington JA (1973) Isolation of a herpesvirus from a pigeon. Aust Vet J 49(1):54-54

29. Phalen DN et al (2017) Prevalence of Columbid herpesvirus infection in feral pigeons from New South Wales and Victoria, Australia, with spillover into a wild powerful owl (Ninox struena). J Wildl Dis 53(3):543-551

30. Woods LW et al (1994) A retrospective study of circovirus infection in pigeons: nine cases (1986-1993). J Vet Diagn Invest 6(2):156-164

31. Hunnam JC et al (2019) The racing pigeon (Columba livia domestica) industry in Victoria, Australia, and epidemiology of a novel group a rotavirus outbreak. Transboundary Emerg Dis 66(5):2058-2066

32. McCowan C et al (2018) A novel group A rotavirus associated with acute illness and hepatic necrosis in pigeons (Columba livia), in Australia. PLoS ONE 13(9):e0203853

Publisher's Note Springer Nature remains neutral with regard to jurisdictional claims in published maps and institutional affiliations. 